\title{
FEATURES OF X-RAY DIAGNOSIS \\ OF THE TEMPOROMANDIBULAR JOINT AT ANOMALIES \\ OF THE RATIO OF DENTAL ARCHES AND POSITION \\ OF THE TEETH ${ }^{1}$
}

\section{Babkina T. M., Storozhchuk Yu. O.}

\section{INTRODUCTION}

Today, pathological changes in the mandibular joint can be the cause or factor influencing the emergence of a number of dental diseases.

The World Health Organization has declared the first decade of the 21st century a "decade of bones and joints". Diseases of the temporomandibular joints are often observed in clinics of surgical, therapeutic, orthopedic dentistry. In most cases, it is difficult to identify the causes and symptoms of these diseases, as well as to establish the causes.

Currently, 98\% of dental patients, according to some authors, have a history of diseases of the temporomandibular joint ${ }^{2,3,4}$. Pathology of the temporomandibular joint ranks third in prevalence among dental problems, caries and periodontal disease - second place.

Questions of diagnosis of TMJ diseases, its structure and function in normal and pathological conditions have been attracting the attention of doctors of various specialties and scientists for many decades. The literature on these issues is extremely contradictory. This indicates a lack of consensus, especially on the pathology, anatomy and function of this joint.

Most authors associate the occurrence of pathological conditions of the temporomandibular joint with a change in occlusion, improperly made prostheses, stress, the presence of a chronic source of infection in the body, improper breathing, diseases of the spine ${ }^{5}$.

${ }^{1}$ The author declares no conflict of interest.

${ }^{2}$ Abakarov, S.I. Age characteristics of the structure of the dental system in patients with distal occlusion / S.I. Abakarov, Yu.M. Malygin, M.Yu. Malygin. Dentistry. 2008. № 1. P. 16-20. 2.

${ }^{3}$ Further significant effects of eldecalcitol on bone resorption markers and bone mineral density in postmenopausal osteoporosis patients having undergone long-term bisphosphonate treatment [Text] / K. Iba et al. J Bone Miner Metab. 2016. № (30). P. 13.

${ }^{4}$ Vlasenko A.Z., Strelkovsky K.M. Technology of making dentures using ceramic and composite materials. Kyiv : Health, 2005.

${ }^{5}$ The sequence of actions of an orthodontist in the correction of dental anomalies complicated by TMJ diseases and parafunctions of the masticatory muscles / R.A. Fadeev et al. Institute of Dentistry. 2015. № 1(66). P. 52-53. 
The positive result of treatment of TMJ diseases largely depends on the knowledge of its anatomy and physiology. The normal structure of the TMJ is the starting point, which makes it possible to correctly assess the structural changes that occur in different types of diseases. Since the structure of the TMJ is widely covered in domestic and foreign literature, it is worth emphasizing only the important features of this joint.

The main teaching of general orthopedics is the interdependence of form and function. The redistribution of functional load on individual facial bones causes a restructuring of the macro- and microstructure, a change in shape. Thus, the preconditions for self-regulation of the mutual arrangement of dental arches and alveolar processes are created. It is a well-known fact that as a result of the habit of chewing food on one side the shape of the articular surfaces of the TMJ changes, a functional asymmetrical position of the lower jaw is established.

There is no doubt that muscle strength and tone change with age and other factors. In addition, there is an anatomical and physiological relationship between the skull, lower jaw, dentition, TMJ, muscles and spine. If one of these elements changes or its function is broken, this process leads to change of all anatomic elements of difficult system.

$\mathrm{X}$-ray examination methods are widely used to identify the causes of joint pathology. Thanks to new radiological technologies, ideas about the morphology of the TMJ in normal and in pathology have expanded. These issues in domestic dentistry are reflected in the works of Professor NA Rabukhina, her colleagues and co-authors. But we are faced with the task of identifying early radiological signs of functional disorders of the TMJ, which in turn require the establishment of a clearer dependence on the condition of other organs of the dental-facial system ${ }^{6}$.

Retained teeth are most often wisdom teeth that have not completely erupted (complete retention) or erupted only partially (partial retention). Often they cause pericoronoritis - an inflammation in the crown of the tooth, which is accompanied by difficulty in opening the mouth and pain.

In cases where the erupted tooth or dental follicle is in an inappropriate place for them, they talk about dystopia, which is mainly observed in a permanent bite. Dystopian teeth can erupt in any part of the mouth, nasal cavity and in the infraorbital margin and orbit. Sometimes they are found in the corner of the lower jaw, the wall of the maxillary sinus. However, the most common anomalies in the location of the teeth in the dental arch.

6 Clinical manifestations of pathology of the temporomandibular joints and masticatory muscles in patients with disorders of occlusion of teeth and dentition / A.V. Lepilin. Saratov Scientific and Medical Journal. 2010. V. 6. № 2. P. 405-410. 


\section{Study goal}

Study of features of X-ray diagnostics of the temporomandibular joint in case of anomalies of the ratio of dental arches and position of teeth.

Materials and methods. All X-ray examinations were performed on a conebeam computed tomography Planmeca ProMax 3D MID with SmartPan system, which allows orthopantomography and teleradiography in the complex.

During 2019, 150 patients with anomalies in the ratio of dental arches and tooth position (distal, mesial occlusion, retained, dystopian teeth - clicks, premolars and 3rd molars) were examined. Patients were divided into 3 age groups.

Group 1: patients aged 9-18 years;

Group 2: patients aged 18-30 years;

Group 3: patients aged 30-45 years.

Healthy patients were not considered.

\section{Results and discussion}

At the initial diagnosis of the 1st group of patients, in the number of 50 (100\%) persons aged 9-18 years, orthopantomography and teleradiography (in lateral projection) were performed. In the anamnesis of all patients were retained and dystopian teeth, medial, distal types of occlusion. 7 (14\%) patients required additional examination to clarify the condition of the temporomandibular joint.

These patients underwent cone-beam computed tomography of the temporomandibular joint in two positions: in normal occlusion and with the widest possible opening of the oral cavity. KPKT-symptoms corresponded to the dysfunction of the temporomandibular joint.

The 2nd group included patients from 18 to 30 years, in the amount of 50 (100\%) people. Of these, 28 (56\%) performed orthopantomography and teleradiography (lateral projection); 22 (44\%) individuals have cone-beam computed tomography of the dentition.

According to these methods of X-ray diagnosis in the examined persons revealed: retained and dystopian teeth, carious cavities, tooth rotation in the dentition, chronic periodontitis, inflammatory changes of the maxillary sinuses and orthopantomography revealed pathological changes in the temporomandibular region. Therefore, 15 (30\%) persons additionally underwent cone-beam computed tomography of the right and left joints in the closed and open position.

3rd group: surveyed 50 (100\%) people aged 30 to 45 years. Of these, $20(40 \%)$ performed orthopantomography and teleradiography (lateral projection); 30 (60\%) people have cone-beam computed tomography of the dentition. In addition to orthodontic pathology, these patients were diagnosed with inflammatory changes in the periodontium, maxillary sinuses.

18 (36\%) persons additionally performed cone-beam computed tomography of the right and left joints in the closed and open position. 
According to the results of the examination, bilateral hypermobility was detected in 9 (18\%) patients, deforming osteoarthritis in $5(10 \%)$, and temporomandibular joint dysfunction in 4 (8\%).

More detailed patient data are given in the table below:

Table 1

Types of TMJ pathology

\begin{tabular}{|c|c|c|c|c|}
\hline \multirow{2}{*}{$\begin{array}{c}\text { Number of } \\
\text { patients }\end{array}$} & \multicolumn{2}{|c|}{ Healthy } & \multicolumn{2}{c|}{ With pathology } \\
\cline { 2 - 5 } & Absolute amount & Interest & Absolute amount & Interest \\
\hline 150 & 38 & $25 \%$ & 112 & $75 \%$ \\
\hline
\end{tabular}

Articular fossae on the skulls and heads of the lower slit for the shape, stepped curvature, the position of their congratulatory axes, which are adapted to each other. There are significant individual indicators of crisis joint tubercle and heads ${ }^{7}$. The peculiar form of bending of the "articular path" of the shoe differs in the individual efficiency of the type of articular sliding. Continuing life with the change of the old dental system, the curvature of the "articular course" was achieved. Changes in the contents of the head in the depression to the front, dosage or back in the absence of anomation of the dental arches indicate pathological changes in the joints.

The TMJ is a closed kinematic circuit because movement in one joint causes movement in another. This joint belongs to the biaxial, as the movement in it occurs on two axes - horizontal and vertical. This joint is incongruent. Concave superficial-articular fossa - does not correspond in size to the convex surface of the mandibular head: the first is two to three times larger than the second. Its congruence is eliminated by a disk and partially by a capsule of a joint.

Maxillofacial anomalies must correspond to morphological substances, aesthetic, occlusal designations, which are observed in sagittal, vertical and transverse application, as well as functionally defined. The high reliability of this mark is studied, which remains the final plan. The scientific literature has indicated that in the present cases, soft tissues were made, which are recommended to create in the skull ${ }^{8}$.

At the same time, the works of some researchers are known ${ }^{9}$, in which on the basis of the X-ray cephalometric method of profile research it is stated

${ }^{7}$ Oral health-related quality of life after orthodontic treatment for anterior tooth alignment : Association with emotional state and sociodemographic factors [Text] / J. Kolenda et al. J Orofac Orthop. 2016. № 2(29). - P. 14.

8 L.A. Determination of myofunctional disorders in children during the period of replacement bite / L.A. Ignatieva, H.X. Khamitova. Clinical dentistry. 2018. № 1(85). P. 61-63.

${ }^{9} \mathrm{X}$-ray anatomy of the temporomandibular joint in patients with dentoalveolar and gnatic forms of mesial occlusion at the stage of planning orthodontic treatment / M.A. Postnikov et al. Modern problems of science and education. 2017. № 6. 
that the correspondence of soft tissue and bone contours is very relative. This allows us to talk about compensation for facial disorders due to the thickness of the soft tissues that cover it. The configuration of the face is largely determined not by the shape and size of its individual parts, but by their interdependent combination ${ }^{10}$.

This, combined with the uneven thickness of the soft tissues of the face, is an explanation for the fact that the anomalies equivalent to dental signs can have a variety of facial features. In her study, she noted a convex profile characteristic of the distal dentition ratio in four patients with mesial occlusion ${ }^{11}$.

Mesial occlusion is an occlusion anomaly characterized by the forward protrusion of the lower dentition relative to the upper with the jaws closed. External manifestations of mesial occlusion are massive, protruding chin, concave chin, concave face profile, sunken upper lip; oral signs include mesial occlusion of molars and canines, direct or reverse incisal occlusion; functional changes - disorders of chewing and speech ${ }^{12}$.

Diagnosis of mesial occlusion is aided by data from anthropometric measurements, teleradiography, orthopantomography, analysis of diagnostic models, and radiography of the TMJ. Among the first and second groups of patients were 26 people who wanted to use braces. In order for the treatment of malocclusion to be successful, we performed all OPTGs before and after the system was installed (see figure 1).

Before orthodontic treatment and after the end of its active period in patients with dental alveolar shortening it is necessary to conduct a comprehensive diagnosis, including anthropometric measurements of the patient's head, diagnostic models of jaws, study of orthopantomologists, head radiographs obtained in lateral projection, and in some cases comp. uterine tomograms, occlusiography data, laboratory and ultrasound examinations. This is necessary to obtain complete diagnostic data and establish the fact of completion of the active period of orthodontic treatment.

That is, the orthodontist needs to know the X-ray diagnosis of TMJ. Such a diagnosis is especially needed by a dentist-surgeon when it comes to removing a retained mandibular tooth, often called a wisdom tooth (see figure 2).

\footnotetext{
${ }^{9}$ Abzalova S.L. Orthodontic treatment of adult patients: analysis of quality aspects / S.L. ${ }^{9}$ Abzalova, A.B. Anokhina. Clinical dentistry. 2018. № 4(88). P. 14-17.

${ }^{11}$ Konnov V.V. Planning the treatment of patients with distal occlusion depending on the anatomical and topographic features of the temporomandibular joints / V.V. Konnov et al. Modern orthopedic dentistry. 2013. № 19. P. 45-48.

12 Orekhova L.Y. Doppler flowmetry as a tool of predictive, preventive and personalized dentistry / L.Y. Orekhova, A.A. Barmasheva. EPMA J. 4(1). 2013. P. 21.
} 


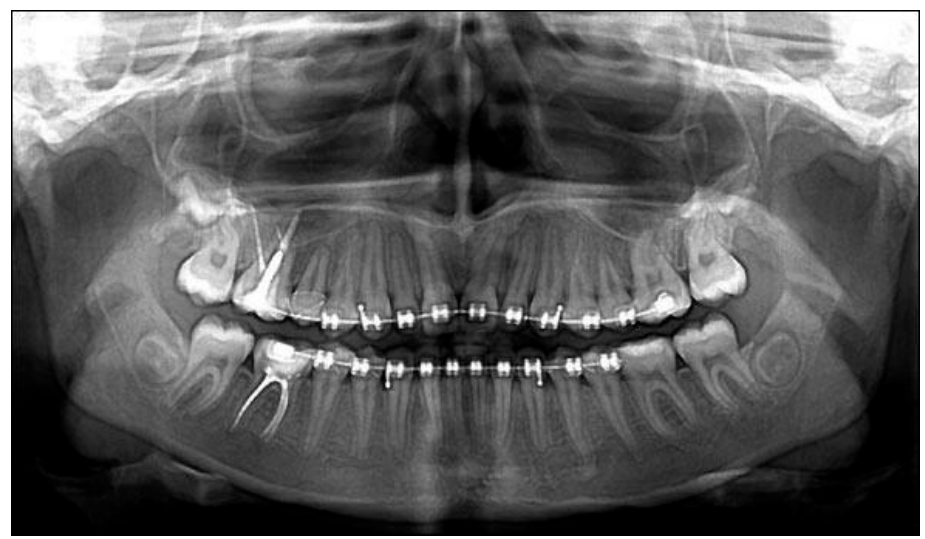

Fig. 1. Radiograph of the patient after installation of the bracket system Radiogram of the patient after installation of the bracket system

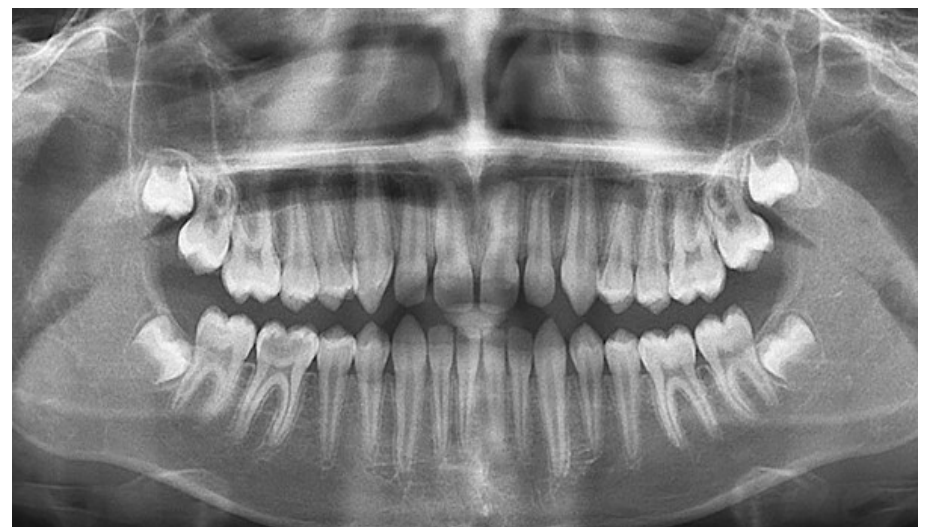

Fig. 2. X-ray of OPTG retained, dystopian teeth (teeth 1.8, 2.8, 3.8, 4.8)

Figure 3 shows a teleradiogram of an 18-year-old patient F, with signs of partial retention of the third molars of the mandible. The Ricketts cephalometric analysis was performed to determine the position of the chin in space, the localization of the upper jaw depending on the convexity of the face, the localization of dental arches in the facial area, the assessment of the soft tissue profile of the face.

At clinical examination, the patient had complaints in the area of the temporomandibular joint, such as pain when chewing, discomfort when opening the mouth. Therefore, the patient underwent cone-beam tomography of the temporomandibular joint in two positions. The results of the presentation in Figure 4. 


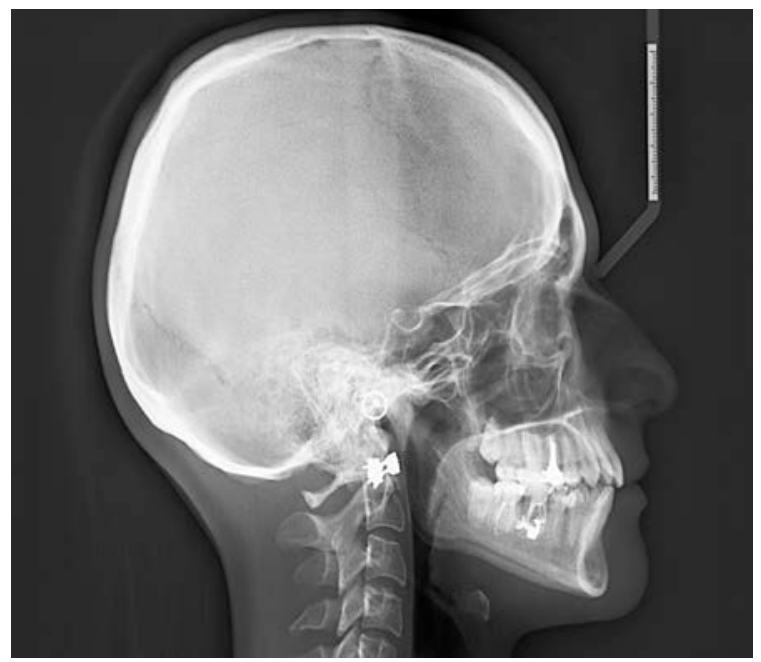

Fig. 3. Clinical case № 1
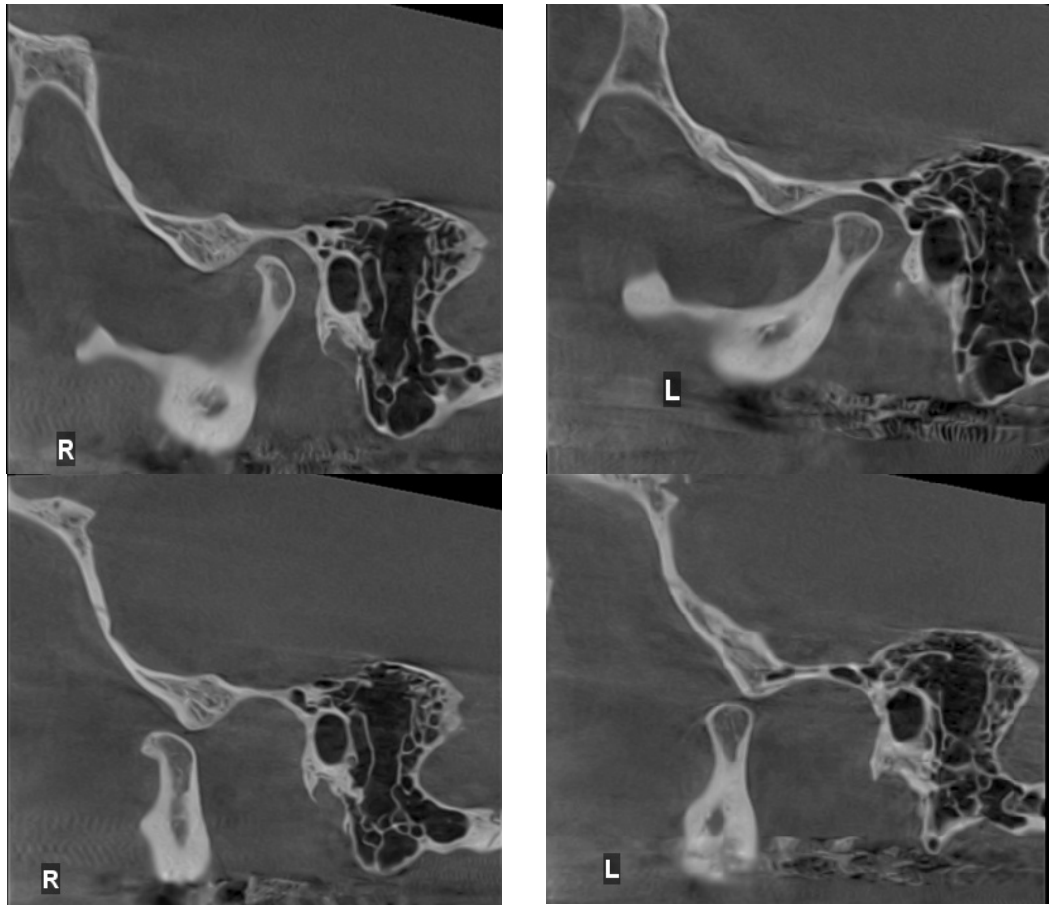

Fig. 4. Clinical case № 1 
Figure 4 shows the axial sections of the CPCT of the mandibular joint with a section thickness of $0.2 \mathrm{~mm}$ in occlusion and with a wide open oral cavity. Analyzing the CBCT data of the temporomandibular joint in occlusion, it was found that the heads of the condyles have an oval shape on the right, on the left - round, slightly flattened in the upper part. On the right, the radiological joint gap is compensatory narrowed in the anterior part, due to the displacement of the joint head to the front. On the left, the radiological joint gap is almost the same throughout, the head is placed centrally.

At the widest possible opening of the oral cavity, the asymmetric position of the condylar processes of the joint is determined: on the right, the head of the condylar process is shifted to the front relative to the articular tubercle by $8.00 \mathrm{~mm}$, the distance between which is $2.20 \mathrm{~mm}$; on the left, the head of the condylar process is shifted to the front relative to the articular tubercle by $6.60 \mathrm{~mm}$, the distance between which is $1.40 \mathrm{~mm}$. This CBCT-symptomatology corresponds to bilateral hypermobility of the temporomandibular joint.

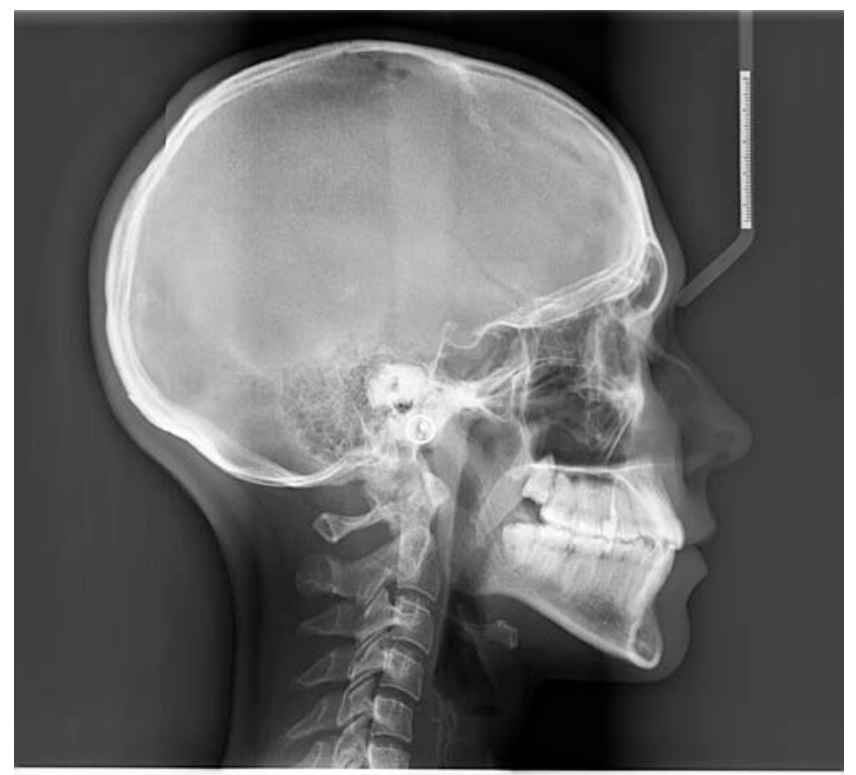

Fig. 5. Clinical case № 2

Figure 5 shows a teleradiography of patient P., 28 years old. According to the examination, partial retention of the third molar of the upper jaw and projection of the tips of the tooth roots into the cavity of the maxillary sinus were revealed; complete retention, dystopia of the third molar of the mandible. The cephalometric analysis was performed according to the 
Ricketts method. Based on clinical data and patient complaints, cone-beam computed tomography of the temporomandibular joint was performed in the closed and open positions.

After CBCT (Figure 6), the following radiological symptoms were detected: occlusion of the head of the condyles has a rounded shape, slightly flattened on the anterior surface on the right, the integrity of the cortical plates of the heads is not violated, osteoporotic bone remodeling on both sides, heads displaced at the ankle buttocks, radiological joint gap is moderately enlarged in the upper part on both sides.

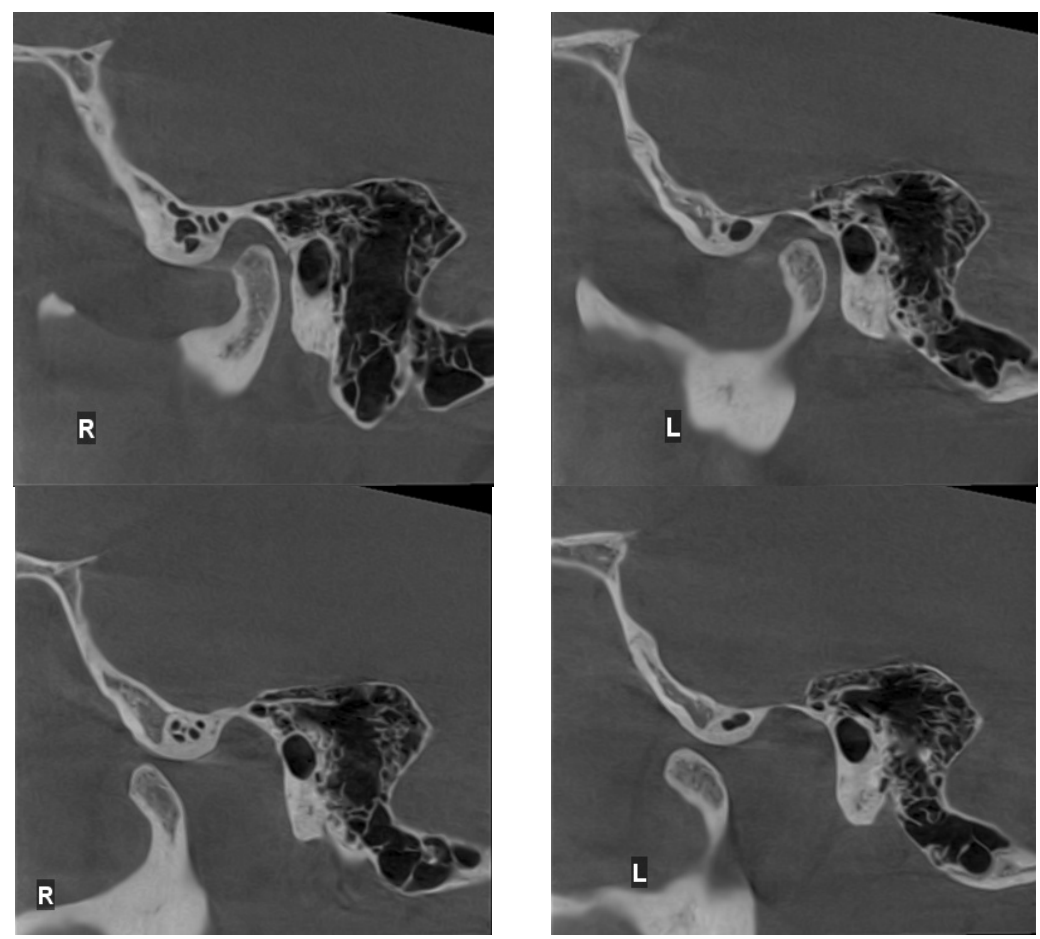

Fig. 6. Clinical case № 2

After CPCT (Figure 6), the following radiological symptoms were detected: occlusion of the head of the condyles has a rounded shape, slightly flattened on the anterior surface on the right, the integrity of the cortical plates of the heads is not violated, osteoporotic bone remodeling on both sides, heads displaced at the ankle buttocks, radiological joint gap is moderately enlarged in the upper part on both sides and initial signs of osteoarthritis. 
Figures 7, 8, 9 show the survey data of patient S. 9 years. The patient was examined on a cone-beam computed tomography scan of two jaws. Patient $\mathrm{S}$. is at the stage of variable occlusion and impaired development of the maxillary area and temporomandibular joint on the right, asymmetry of the maxillofacial area, multiple carious cavities, delayed eruption of teeth. For detailed visualization and assessment of the anatomical structures of the temporomandibular joint, CPCT was performed in occlusion.

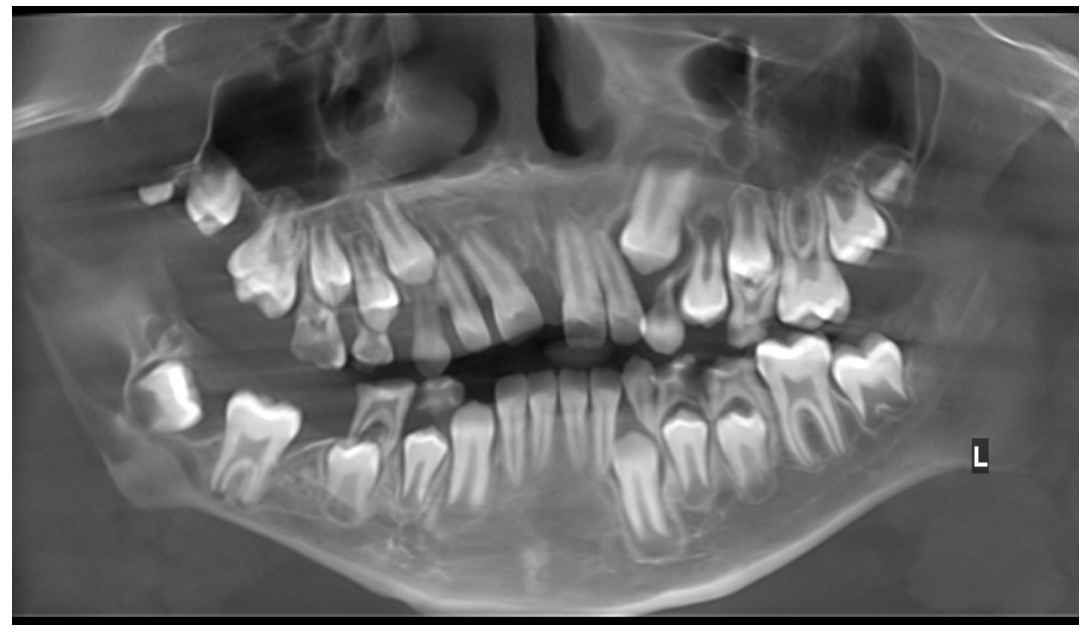

Fig. 7. Clinical case № 3. Orthopantomogram-2d reformat from cone-beam computed tomography
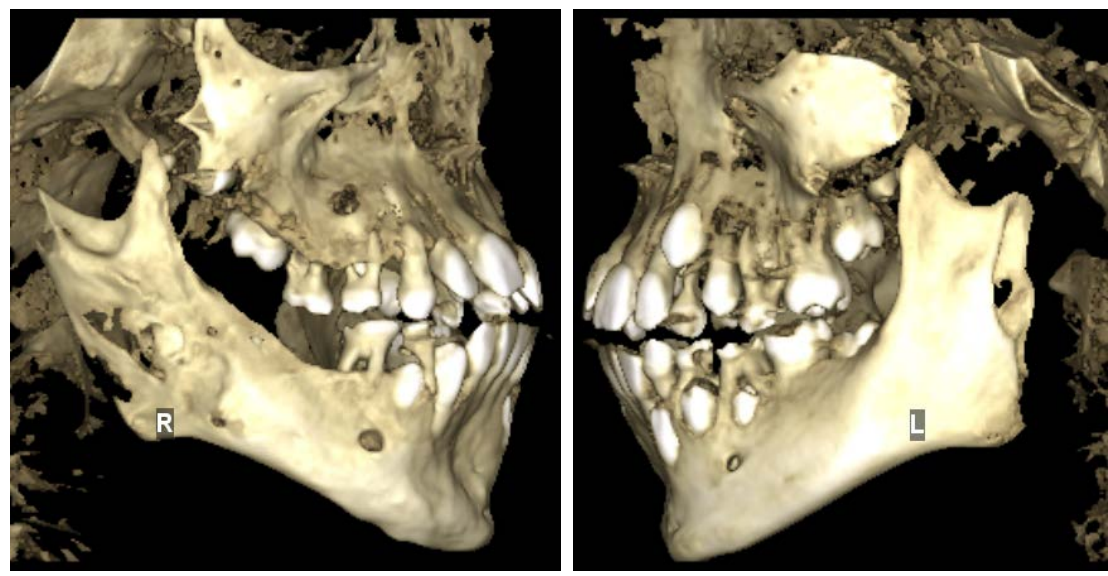

Fig. 8. Clinical case № 3. 3D rendering of the upper and lower jaws 

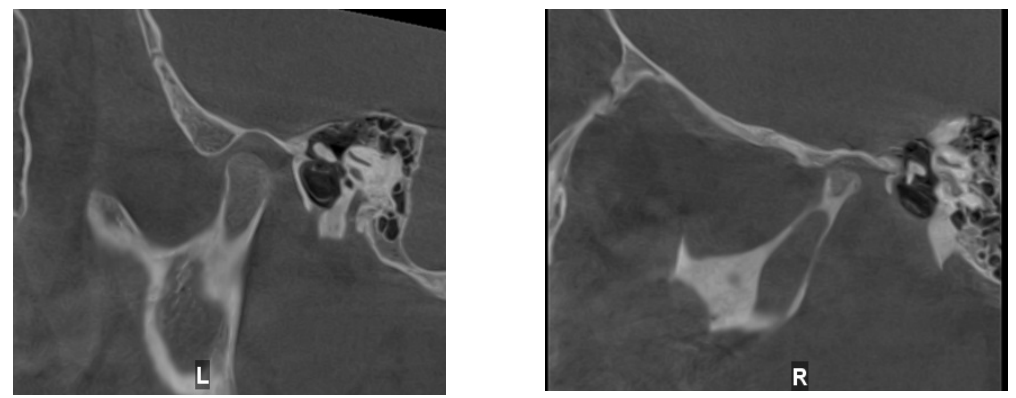

Fig. 9. Clinical case №3. Sagittal sections of the temporomandibular joint

Figures 10,11, 12, 13 show the results of the examination of the patient $\mathrm{T}$. 32 years old. The patient underwent primary diagnosis of two jaws on conebeam tomography and revealed complete retention, tooth dystopia 1.8, 2.8, 4.8, partial retention and dystopia of tooth 3.8., Congestion of the front group of mandibular teeth, deep carious cavity of tooth 3.4. On coronal and sagittal sections the projection of tops of roots of teeth 1.8, 2.8 in a cavity of maxillary sinuses is well visualized.

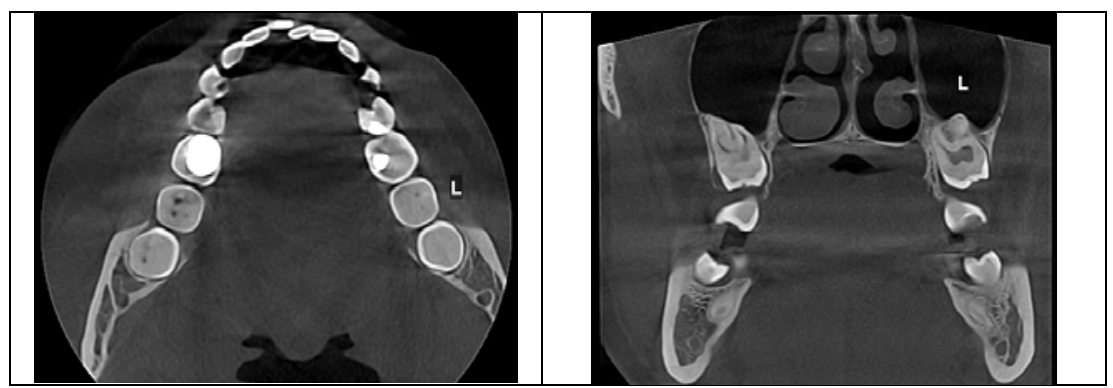

Fig. 10. Clinical case № 4

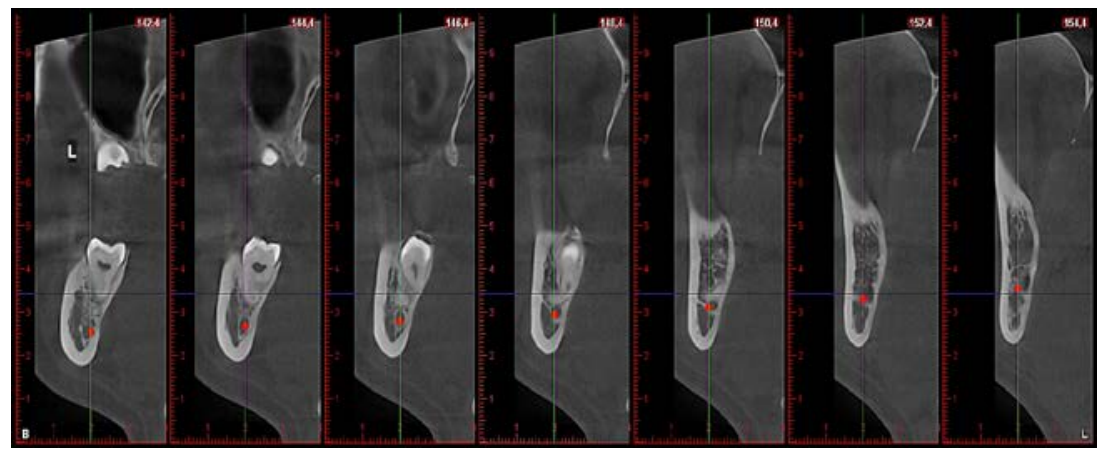

Fig. 11. Clinical case № 4 


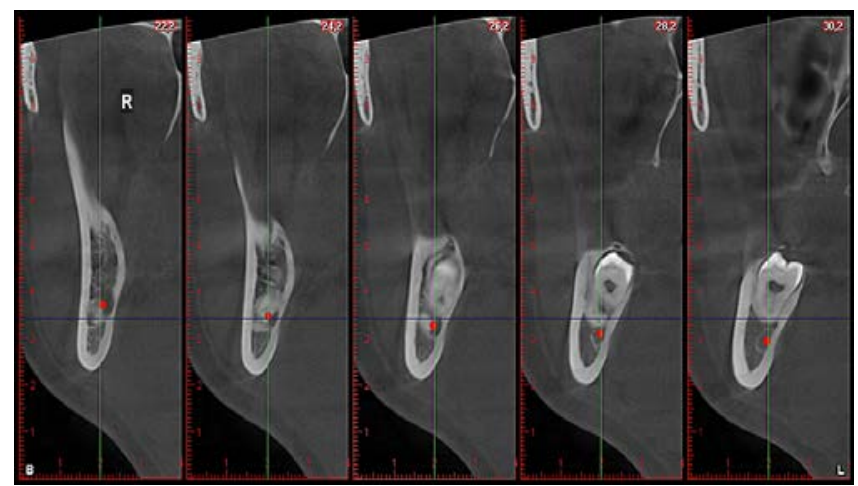

Fig. 12. Clinical case № 4
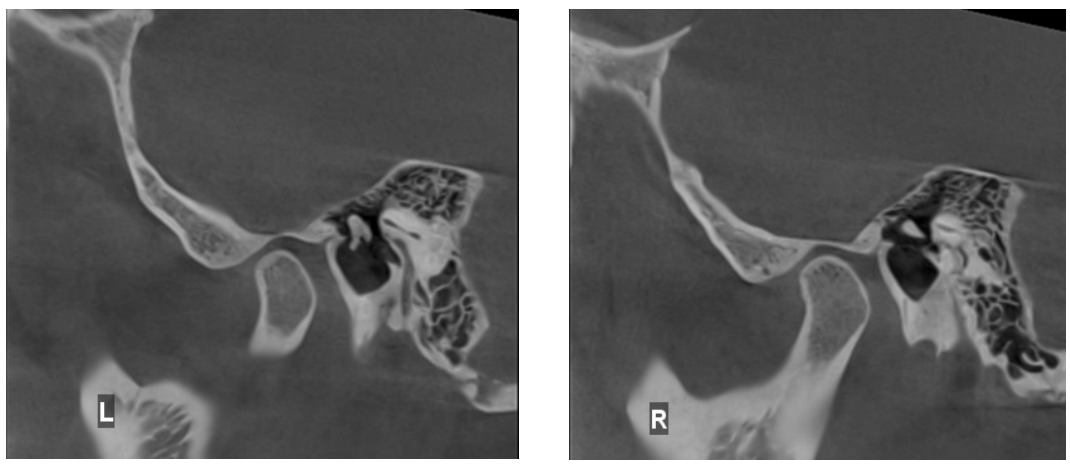

Fig. 13. Clinical case № 4

Figure 14 shows the cross sections of the CBCT on which the position and ratio of the tops of the roots of the retentiated, dystopian teeth 3.8, 4.8 to the mandibular canal are clearly determined. Only CBCT gives detailed information about such an important topographic correlation of the anatomical structures of the dentofacial system.

Figure 15 shows the sagittal sections of the CBCT of the temporomandibular joint, according to which a moderate decrease in the radiological joint space, bilateral displacement of the condylar process heads anteriorly in the articular cavities is determined, which may indicate joint dysfunction.

Figure 16 shows the teleentgenogram of patient K., 35 years old. After evaluating the initial study and the patient's clinical data, an additional study was conducted on a cone beam computed tomography scanner. The area of study of the temporomandibular joint in occlusion and with the widest possible open oral cavity. 


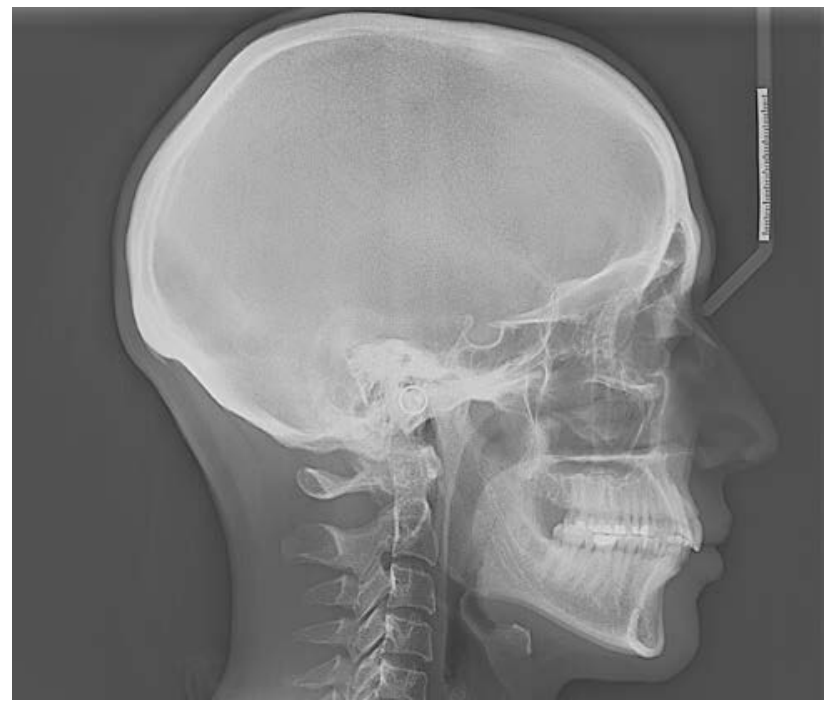

Fig. 14. Clinical case № 5
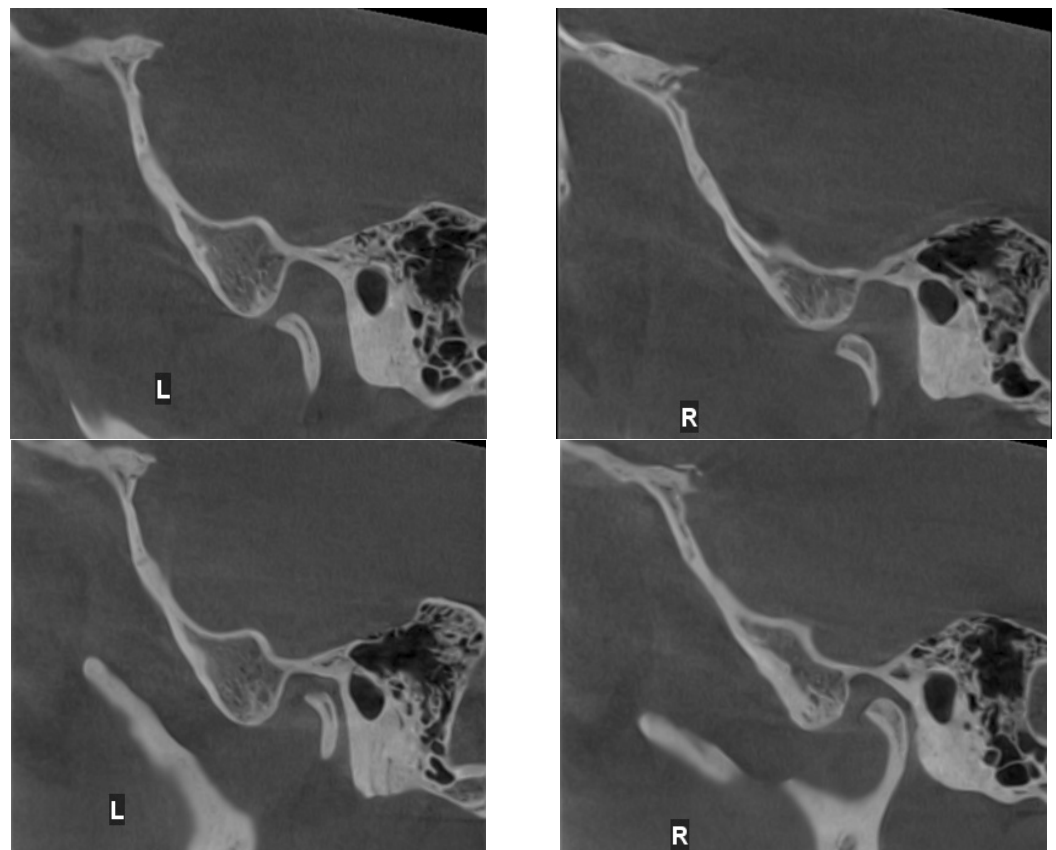

Fig. 15. Clinical case № 6 
Figures 15 and 16 show sagittal sections with a thickness of $0.2 \mathrm{~mm}$. When evaluating the TMJ in occlusion of the head of the condylar processes of the "hook-shaped" shape, which is flattened on the right on the front surface, on the left - in the upper section; osteosclerosis of the locking plates of the heads of the joint, osteoporotic bone remodeling, displacement of the heads in the articular cavity posteriorly and downward. With a wide opening of the oral cavity, the displacement of the heads of the condylar processes anteriorly in the articular cavities is determined (the heads of the condylar processes are in contact with the posterior slope of the articular tubercle). CBCT symptomatology corresponds to bilateral dysfunction of the temporomandibular joint, osteosclerosis.
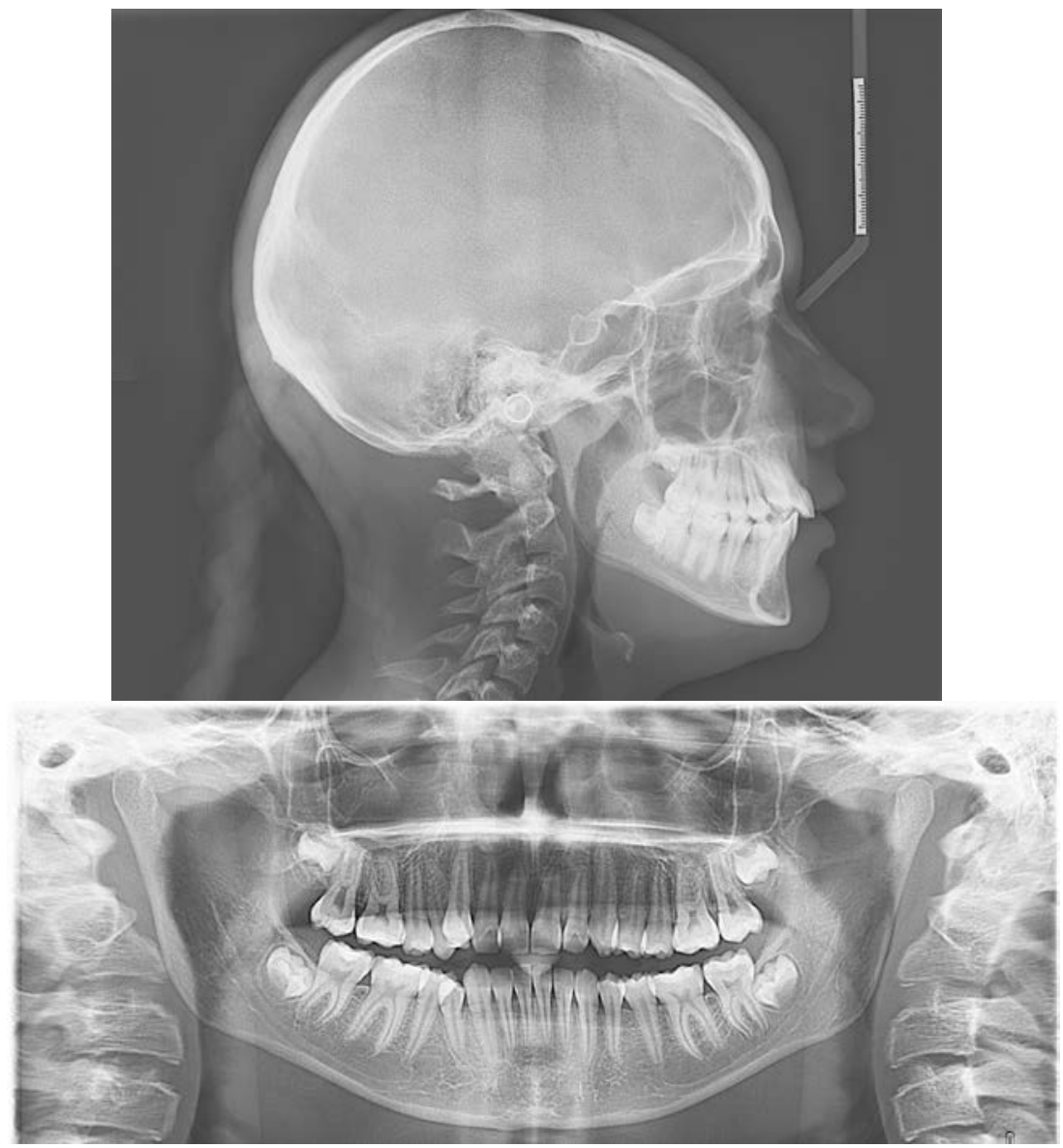

Fig. 16. Clinical case № 7 
Figure 16 shows the study of a patient At 19 years old. On the otropantogram, the rudiments of teeth 1.8, 2.8, 3.8, 4.8, in the alveolar processes of the upper and lower jaw, which are closely adjacent to the roots of the teeth $1.7,2.7,3.7,4.7$, are visualized. Tooth 1.4 is rotated distally and displaced vestibularly in the dentition, as a result of which "presses" on the coronal part of the tooth 1.3 . Tooth 2.3 rotated distally in the dentition by 90 degrees. 4.4 the tooth is inclined distally, the crown part of which is closely adjacent to the vestibular surface of the tooth crown 4.5. Marked changes in the position of the above teeth are due to the presence and location of third molars of the upper and lower jaw.

\section{CONCLUSIONS}

The frequency of dental anomalies, their prevalence varies according to various authors up to $80 \%$. The presence of dentoalveolar anomalies leads to morphological, functional and aesthetic disorders of the maxillofacial area and are reflected in the activities of other organs and systems of the body.

The success of treatment of dental anomalies is largely determined by the accuracy of diagnosis. The three-dimensional reconstructions obtained during CPCT provide more accurate information about the anatomical structure of the temporomandibular joint, the position of the heads of the lower jaw in the articular cavity. These elements are important in the presence of morphological, functional disorders.

Cone-beam computed tomography of the temporomandibular joint has a higher accuracy in comparison with orthopantomography and teleradiography. On sections, it is possible to accurately detect changes such as changes in the cortical plate (erosion), bone growths (osteophytes), which are manifestations of restructuring of the articular heads, cystic formations, degenerative changes in the anatomical elements of the joint, narrowing of the joint gap, ankylosis.

\section{SUMMARY}

There are many dental-maxillary anomalies of development, but the most relevant is the violation of the ratio of dental arches. The question of choosing the most informative and safe radiation method for diagnosing this pathology remains debatable. In the work, known methods for diagnosing VRDA with abnormalities are considered, the ratio of dental arches and tooth position. The frequency of detection of these abnormalities in various age groups of patients is analyzed and recommendations on the plan of diagnostic measures for each of the age groups are given.

Study goal. To assess the specifics of the use of radiological methods of diagnosis of the temporomandibular joint among orthodontic patients and determine the correctness of the use of this technique. 
Materials and methods. X-ray diagnosis was performed in 150 patients with anomalies of the ratio of dental arches and the position of the teeth in three age groups 9-18, 18-30, 30-45 years. Radiological methods of research were carried out on a Planmeca ProPax 3D MID cone-beam computed tomograph with SmartPan system.

Results. Assessing the obtained data, it was found that cone-beam computed tomography, in contrast to other methods of X-ray diagnostics, provides more diagnostic information that is necessary for making a diagnosis and the right treatment method.

Conclusions. The frequency of dental anomalies, their prevalence varies according to various authors up to $80 \%$. The presence of ZAU leads to morphological, functional and aesthetic disorders of the maxillofacial area and are reflected in the activities of other organs and systems of the body.

The success of treatment of dental anomalies is largely determined by the accuracy of diagnosis.

\section{REFERENCES}

1. Abakarov S.I. Age characteristics of the structure of the dental system in patients with distal occlusion / S.I. Abakarov et al. Dentistry. 2008. № 1. P. 16-20.

2. Further significant effects of eldecalcitol on bone resorption markers and bone mineral density in postmenopausal osteoporosis patients having undergone long-term bisphosphonate treatment [Text] / K. Iba et al. J Bone Miner Metab. 2016. № (30). P. 13.

3. Vlasenko A.Z., Strelkovsky K.M. Technology of making dentures using ceramic and composite materials. Kyiv : Health, 2005.

4. The sequence of actions of an orthodontist in the correction of dental anomalies complicated by TMJ diseases and parafunctions of the masticatory muscles / R.A. Fadeev et al. Institute of Dentistry. 2015. № 1(66). P. 52-53.

5. Clinical manifestations of pathology of the temporomandibular joints and masticatory muscles in patients with disorders of occlusion of teeth and dentition / A.V. Lepilin et al. Saratov Scientific and Medical Journal. 2010. V. 6. № 2. P. 405-410.

6. Oral health-related quality of life after orthodontic treatment for anterior tooth alignment : Association with emotional state and sociodemographic factors [Text] / J. Kolenda et al. J Orofac Orthop. 2016. № 2(29). P. 14.

7. Ignatieva L.A. Determination of myofunctional disorders in children during the period of replacement bite / L.A. Ignatieva, H.X. Khamitova. Clinical dentistry. 2018. № 1(85). P. 61-63. 
8. X-ray anatomy of the temporomandibular joint in patients with dentoalveolar and gnatic forms of mesial occlusion at the stage of planning orthodontic treatment / M.A. Postnikov et al. Modern problems of science and education. 2017. № 6.

9. Abzalova S.L. Orthodontic treatment of adult patients: analysis of quality aspects / S.L. Abzalova, A.B. Anokhina. Clinical dentistry. 2018. № 4(88). P. 14-17.

10. Konnov V.V. Planning the treatment of patients with distal occlusion depending on the anatomical and topographic features of the temporomandibular joints / V.V. Konnov et al. Modern orthopedic dentistry. 2013. № 19. P. 45-48.

11. Orekhova L.Y. Doppler flowmetry as a tool of predictive, preventive and personalized dentistry [Text] / L.Y. Orekhova, A.A. Barmasheva. EPMA J. 4(1). 2013. P. 21.

\section{Information about authors:} Babkina T. M.,

Doctor of Medical Sciences, Professor, Radiology Department of Radiology Shupyk National Medical Academy of Postgraduate Education 9, Dorohozhytska str., Kyiv, 04112, Ukraine Storozhchuk Yu. O., Graduate Student, Assistant of the Department of Radiology Shupyk National Medical Academy of Postgraduate Education 9, Dorohozhytska str., Kyiv, 04112, Ukraine 